ORIGINAL ARTICLE

\title{
Standard pulse oximeters can be used to monitor respiratory rate
}

\author{
P Leonard, T F Beattie, P S Addison, J N Watson
}

See end of article for authors' affiliations

Correspondence to: Dr P Leonard, Accident and Emergency Department, Royal Hospital for Sick Children, Sciennes Road, Edinburgh EH9 1LF, Scotland: paul_leonard@ blueyonder.co.uk

Accepted for publication 10 January 2003
Background: One of the most important limitations of standard pulse oximeters is the inability to detect changes in respiratory rate until oxygenation is affected. This study sought to determine if analysis of the plethysmogram by wavelet transforms would enable the determination of changes in respiratory rate at an earlier stage.

Methods: Ten healthy adult volunteers were monitored, breathing at baseline and predetermined respiratory rates, using a standard pulse oximeter. Photo-plethysmograms captured in an attached lap top computer were then analysed using wavelet transforms.

Results: Determination of baseline respiratory rate and subsequent changes including apnoea were easily identified.

Comment: Wavelet transforms permit the accurate determination of respiratory rate by a standard pulse oximeter.
$\mathrm{P}$

ulse oximetry provides a non-invasive and comparatively inexpensive method of continuously monitoring oxygenated haemoglobin in blood based on the differential light absorption properties of oxygenated and deoxygenated haemoglobin. It provides an accurate measure of both heart rate and oxygen saturation ${ }^{1}$ and is widely used in accident and emergency (A\&E) departments to monitor patients at risk of hypoxia. Clinicians are familiar with the appearance of the wave form (plethysmogram) produced by the pulse oximeter, but only use it to determine if the oximeter is working correctly. ${ }^{2}$ We report on the discovery that wavelet signal analysis techniques permit the determination of the respiratory rate from the plethysmogram produced by a standard pulse oximeter.

Plethysmogram traces were obtained from 55 critically unwell children as part of a larger study, and were analysed using wavelet transforms. Wavelet transforms are an ideal tool for analysing biological signals as they allow a signal to be decomposed such that both the frequency characteristics and the location of particular features in a time series may be highlighted simultaneously. ${ }^{3}$ This allows the technique to rapidly identify changes in a signal at the same time as allowing the removal of artefact (for example, due to movement). These benefits have resulted in wavelet transforms being increasingly used for analysing signals from a wide variety of medical devices. ${ }^{45}$ While examining these signals it became apparent that a low frequency signal corresponding to the respiratory rate was detectable within the plethysmogram (fig l) and we wished to determine if this technique could be used to accurately determine respiratory rate and could rapidly detect changes in respiratory rate.

\section{METHODS AND RESULTS}

Ten healthy adult volunteers were recruited, and baseline plethysmograms were obtained by monitoring them for a period of one minute using a Nellcor 100 monitor connected via a PCMCIA-PCM-DAS16D/16 card to a standard Pentium PC lap top computer. They were then asked to breathe at a rate of exactly 30 breath/min for one minute, using a stopwatch to pace themselves, then to reduce their respiratory rate to 15 breath/min for a further minute, before stopping breathing for as long as possible.
Plethysmograms were analysed using wavelet transforms, and permitted accurate determination of the base line respiratory rate in all volunteers. Wavelet analysis also permitted the rapid detection of the halving of respiratory rate (fig 2) and of apnoea.

\section{DISCUSSION}

Conventional pulse oximeters currently do not provide any information about the respiratory rate of a patient and apnoea will only be recognised when hypoxia has occurred, giving little time to institute corrective measures. Monitoring of respiratory rate is currently performed manually by nursing staff, by measuring changes in thoracic impedance via ECG leads or by monitoring carbon dioxide levels in expired air (capnography).

Each of these methods has disadvantages. Manual measurements are intermittent and require a significant amount of nursing time if they are to warn of sudden changes in respiratory rate. Monitoring of thoracic impedance is prone to error from movement artefact. Capnography requires additional, often expensive, equipment and a way of positioning the probe in the exhaled airflowthis is expensive and difficult to achieve with the vast majority of A\&E patients who are self ventilating and do not require oxygen therapy.

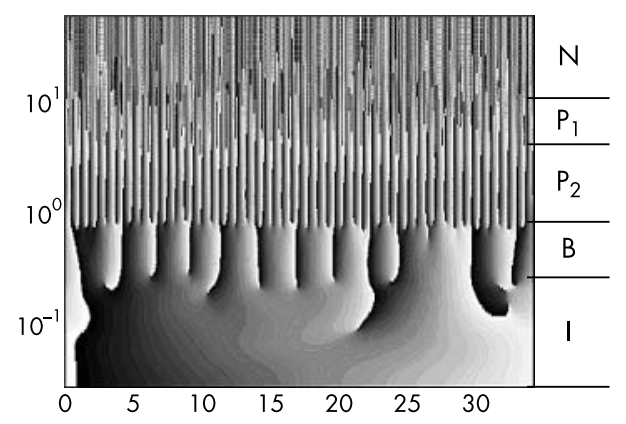

Figure 1 Wavelet plot showing easily identified low frequency feature in region $\mathrm{B}$ (breathing) and how this is easily separated from noise/ artefact $(N)$, and pulse rate $\left(P_{1}\right.$ and $\left.P_{2}\right)$. 
A

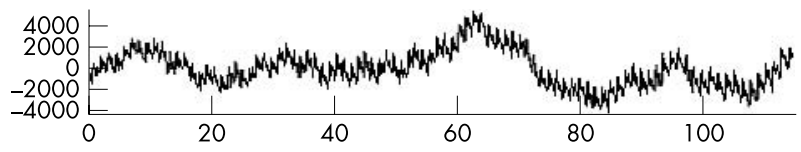

B

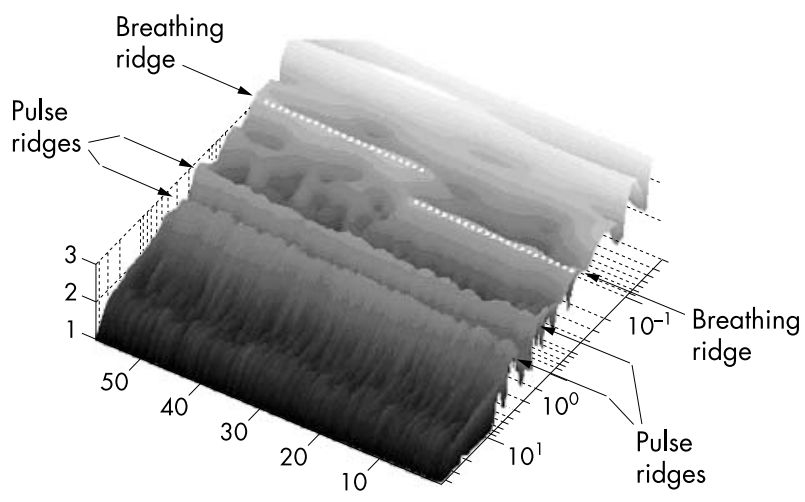

Figure 2 Pulse oximeter tracing (A) and associated Modulus plot (B) showing extraction of respiratory tracing (breathing ridge) and easily identifiable change when respiratory rate halved.

While specialist probes have been used to identify respiratory rates form plethysmograms in neonates ${ }^{6}$ we believe that this is the first time wavelet transforms have been used to detect respiratory signals in plethysmograms obtained from standard pulse oximetry equipment. As the initial discovery was made on paediatric traces taken in an acute clinical setting, and our small adult study has shown that respiratory signals are detectable in adults we expect that this technique is applicable to all age groups and will cope with the artefact, etc, created during standard clinical practice.

We are confident that wavelet techniques will provide a comparatively inexpensive and robust way of monitoring respiratory rate with only minor adaptations to current technology. We are currently working on a real time algorithm that we hope can be incorporated in future pulse oximeters. This will greatly improve the role of the pulse oximeter, permitting the rapid detection and correction of apnoea or bradypnoea before any change in the oxygen saturation.

\section{Authors' affiliations}

P Leonard, T F Beattie, Department of Accident and Emergency Medicine, The Royal Hospital for Sick Children, Edinburgh, Scotland

P S Addison, Faculty of Engineering and Computing, Napier University, Edinburgh, Scotland

J N Watson, Cardiodigital Ltd, Edinburgh, Scotland

\section{REFERENCES}

1 Trafford J, Lafferty K. What does the photoplethysmograph measure? Med Biol Eng Comput 1984;22:479-80.

2 Moyle JT. Pulse oximetry. London: BMJ Publishing Group, 1994.

3 Mallat S. A wavelet tour of signal processing. San Diego: Academic Press, 1998.

4 Bracic $M$, Stefanovska A. Wavelet-based analysis of human blood flow dynamics. Bull Math Biology 1998;60:919-35.

5 Addison PS, Watson JN, Clegg GR, et al. Finding coordinated atrial activity during ventricular fibrillation using wavelet decomposition. IEEE Engineering in Medicine and Biology 2002;21:58-65.

6 Vegfors M, Lindberg L, Pettersson H, et al. Presentation and evaluation of a new optical sensor for respiratory rate monitoring. International Journal of Clinical Monitoring and Computing 1994;11:151-6. 